\title{
Editorial
}

Theme: Advancements in Dissolution Testing of Oral and Non-Oral Formulations

Guest Editor: Sandra Klein

\section{Advancements in Dissolution Testing of Oral and Non-oral Formulations}

\author{
Sandra Klein ${ }^{1,2}$
}

Received 11 July 2019; accepted 12 July 2019; published online 25 July 2019

It was as early as in the late nineteenth century that the first dissolution test was performed by Arthur A. Noyes and Willis R. Whitney, who studied the dissolution of substances in their own solutions (1). These experiments were for sure not intended to determine the quality of pharmaceutical dosage forms, but fundamental for modern pharmaceutical dissolution testing. Noyes and Whitney were able to explain the theoretical foundations of dissolution testing, but it took more than 50 years from then, until with the rotating basket apparatus in 1970 the first official dissolution apparatus was incorporated into the US Pharmacopeia (USP). During the 1970s, there were 12 official dissolution tests using baskets in USP monographs. The paddle apparatus followed shortly thereafter (2). During the following decades, dissolution testing has assumed more and more importance. Advances included the development of new instruments, novel dissolution media, and methods. Significant progress was made in the use of biorelevant dissolution media and the simulation of in vivo hydrodynamics for estimating in vivo drug release of oral dosage forms. Moreover, a significant number of novel methods were developed for dosage forms that are not administered orally. Currently, dissolution testing is routinely used to provide critical in vitro drug release information for quality control (QC) purposes, can be a promising tool for predicting in vivo drug release, and plays an essential role in the Biopharmaceutics Classification System (BCS)-based biowaiver approach, i.e., in waiving in vivo bioavailability (BA) studies by using in vitro dissolution profiles as a surrogate basis for the decision as to whether the two pharmaceutical products are equivalent.

This theme issue on advancements in dissolution testing of oral and non-oral formulations is dedicated to give an update on, but also to critically review current biorelevant and quality control methods, to present results from ongoing research in the

Guest Editor: Sandra Klein

${ }^{1}$ Department of Pharmacy, Institute of Biopharmaceutics and Pharmaceutical Technology, Center of Drug Absorption and Transport, University of Greifswald, 3 Felix Hausdorff Street, 17489, Greifswald, Germany.

${ }^{2}$ To whom correspondence should be addressed. (e-mail: Sandra.Klein@uni-greifswald.de) development of bio-predictive test methods for novel dosage forms and shall also provide guidance on how to implement results from in vitro testing into the design of physiologically based pharmacokinetic (PBPK) models.

The ability to properly discriminate between drug products of different quality was always an essential requirement for a dissolution method. However, whereas for a long time a method was regarded as appropriate when it could indicate differences among changes in critical material attributes and critical process parameters applied in the manufacture of the dosage form, there are ongoing efforts to strengthen the linkage to the in vivo performance of the dosage form by use of improved media, apparatus, and PBPK (2).

As discussed in the mini-review on "Power of the Dissolution Test in Distinguishing a Change in Dosage Form Critical Quality Attributes", if a dissolution test is both discriminatory and in vivo predictive, it is a powerful tool that alerts that there may be bioinequivalent batches produced and helps to avoid that patients receive drugs that are not fully efficacious (2).

Since the start of the twenty-first century, the development of physiologically relevant and in vivo predictive dissolution methods, also referred to as biorelevant or bio-predictive dissolution methods, is a main focus of research in the field of in vitro dissolution testing. For oral dosage forms, a biorelevant dissolution method should address all essential parameters that can affect drug release in the human gastrointestinal (GI) tract after drug administration in the fasted or the fed state. The main focus of the first biorelevant dissolution methods was set on simulating the composition of GI contents after oral drug administration in fasted and fed state dosing conditions according to a typical clinical study protocol (3). Since the introduction of the first set biorelevant media that mainly addressed conditions in the stomach and upper small intestine, these media have been further fine-tuned and nowadays a variety of biorelevant media addressing the intraluminal composition at different sites in the GI tract, but also conditions at other sites of drug administration are available.

In many cases, the composition of physiological fluids is just one aspect relevant for dissolution and drug release. In vivo drug release from oral dosage forms can be affected 
by various physiological factors such as GI transit time, hydrodynamics, shear forces, and stress events. This becomes of particular importance when assessing drug release of extended-release (ER) formulations (4). The selection of suitable in vitro methods should therefore be based on a thorough understanding of human GI physiology, but also of drug and formulation properties. The review article "Physiological Considerations and In Vitro Strategies for Evaluating the Influence of Food on Drug Release from ExtendedRelease Formulations" focuses on in vitro methods that can be applied to evaluate the effect of food intake on drug release from ER drug products during preclinical formulation development and by presenting several case studies demonstrates that the selection of appropriate biorelevant in vitro methods can be extremely useful for understanding in vivo drug release from ER products and for forecasting formulation-associated risks, such as dose dumping, in early stages of formulation development (5).

Reproducible retention of dosage forms in the stomach is a difficult task. To enhance gastric residence of so-called gastroretentive formulations, these dosage forms are often coadministered with food. However, food can affect in vivo drug release and oral BA by several pathways. One physiological parameter that can be strongly affected by food intake is GI motility. Postprandial motility can significantly contribute to the failure of gastroretentive dosage forms. The research paper "Influence of Postprandial Intragastric Pressures on Drug Release from Gastroretentive Dosage Forms" presents results from a study investigating the influence of simulated physiological postprandial pressure conditions on drug release from gastroretentive systems. When screening in vitro drug release of these formulations, results from standard dissolution tests using the paddle apparatus displayed controlled drug release from both dosage forms tested. By contrast, results obtained in the different physiologically relevant in vitro tests revealed a highly pressuresensitive drug release behavior of the dosage forms tested, indicating that in vivo a controlled release behavior is rather unlikely (6). Results of these experiments highlight the importance of properly addressing in vivo conditions and help to explain why in the past so many gastroretentive formulations failed in first-in-man studies.

Also in the fasted state, GI parameters relevant for in vivo dissolution and drug release can show a high inter- and intraindividual variability. For some dosage forms, particularly for those with $\mathrm{pH}$-dependent drug release, these variabilities can be the source of BA issues. The study on assessing the impact of physiological variability in fasted GI $\mathrm{pH}$ profiles on diclofenac sodium release from matrix tablets ("The Influence of Simulated Fasted Gastrointestinal pH Profiles on Diclofenac Sodium Dissolution in a Glass-Bead Flow-Through System") represents an attempt for estimating in vivo drug release of a weakly acidic drug compound from an ER formulation in individual subjects (7) and is a simple precursor method of more advanced individualized in vitro methods that target on a precise prediction of the in vivo performance or an in vitro-in vivo correlation (IVIVC), respectively $(8,9)$.

In vitro dissolution data from properly designed in vitro experiments can provide a biorelevant input for in vivo predictive in silico models. The article "Physiologically Based Absorption Modeling of Salts of Weak Bases Based on Data in Hypochlorhydric and Achlorhydric Biorelevant Media" discusses how in vitro dissolution and precipitation profiles obtained with a biorelevant gastrointestinal transfer (BioGIT) system were successfully used for informing a computational model for estimating plasma profiles of poorly soluble weakly basic model compounds in regard to the precipitation kinetics in patients with elevated gastric $\mathrm{pH}$ (10). In future experiments, this approach can be applied to other compounds of the same kind and may help to establish in silico models for predicting the in vivo performance of poorly soluble weakly basic drugs in hypo- or achlorhydric patients.

All cited contributions point towards a paradigm change in in vitro dissolution testing. When the dissolution test is no longer limited to a pure QC tool, but should provide a reliable prediction of the in vivo performance of oral dosage forms in different patients, individual GI physiology and the dosing conditions need to be properly addressed in the in vitro setup. Therefore, it is likely that in the future, we will see an increasing number of individualized or patientspecific in vitro test designs that will hopefully help to improve safety and efficacy of oral drug products for the target patient groups. Overall, the number and variety of biorelevant dissolution test methods is expected to increase, since in the recent past, various novel dosage forms for both oral and non-oral drug administration for which such test methods have yet to be developed were introduced.

Oromucosal film preparations represent a novel formulation type that has gained popularity in pharmaceutical research and development. To date, an official test method for these formulations is not available. The research paper "Novel Dissolution Method for Oral Film Preparations with Modified Release Properties" describes the development of a novel flow-throughcell-based dissolution method and a subsequently performed study targeted on better understanding how variation of different test parameters, such as sample holder, flow rate, and media composition affects the dissolution performance of an oromucosal film as well as the discriminatory power of the method (11). A systematic study aimed to study how geometry of the dissolution vessel, media volume, and composition might contribute to the variation in drug release from a novel delivery system is reported in the article "Influence of Dissolution Vessel Geometry and Dissolution Medium on In Vitro Dissolution Behaviour of Triamterene-Coated Model Stents in Different Test Setups." The main goal of this study was to determine appropriate in vitro test conditions for drug-eluting stents, i.e., novel drug/device combination products (12). Results from these two studies indicate that a proper method development requires a thorough understanding of the parameters that affect the dissolution process of the drug (product) to be tested. A parameter that may also play an important role in this regard is the viscosity of the dissolution medium which is influenced by the media composition. The report on "Understanding the Potential for Dissolution Simulation to Explore the Effects of Medium Viscosity on Particulate Dissolution" describes the application of dissolution simulation methods to exploring the effects of slight changes in media viscosity on particulate dissolution in the flowthrough cell and the paddle apparatus and provides a nice insight into the chances and limitations of simulations in better understanding particle dissolution (13).

Several research papers in this issue are dedicated to novel dissolution methodologies comprising miniaturized methods that are of particular interest in early formulation screening where typically only a small amount of the active pharmaceutical 
ingredient (API) may be available. Surface dissolution ultraviolet (UV) imaging is a novel technique for assessing drug dissolution in a very small scale. The paper "Surface Dissolution UV Imaging for Investigation of Dissolution of Poorly Soluble Drugs and Their Amorphous Formulation" describes the application of this technique in investigating the dissolution properties of poorly soluble drugs from their pure form and their amorphous formulations under physiological relevant conditions (14). The article "A Modified In Situ Method to Determine Release from a Complex Drug Carrier in Particle-Rich Suspensions" addresses light scattering, a common issue in in situ UV absorption measurement in many dissolution experiments, and proposes a modification of the analytical setup that enables in situ drug release assessment in particle-rich suspensions (15).

Another novel in vitro approach comprises the combination of dissolution and permeation studies for predicting oral drug absorption of poorly soluble drugs. The article "Using $\mathrm{pH}$ Gradient Dissolution with In-Situ Flux Measurement to Evaluate Bioavailability and DDI for Formulated Poorly Soluble Drug Products" describes an in vitro dissolution-permeation device that had been applied for studying dissolution and permeation of final clinical dosage forms of three poorly soluble, but highly permeable drugs. Two of the three formulations were also evaluated in vivo and the obtained in vitro dissolution and flux results were in good correlation with dog and human pharmacokinetic $(\mathrm{PK})$ data indicating that results obtained with the novel in vitro setup were predictive for the in vivo performance of the respective dosage forms.

In vitro dissolution and permeation testing of a particular group of non-oral drug formulations are discussed in "In Vitro Drug Dissolution/Permeation Testing of Nanocarriers for Skin Application: a Comprehensive Review." The focus of this review article is set on discussing in vitro test methods for topical and transdermal nanocarriers that have been designed to modulate the propensity of drug release, drug penetration into the skin, and permeation into the systemic circulation. The review highlights that for designing both biorelevant in vitro models that can be applied for predicting the clinical performance of topical and transdermal nanocarriers and robust and discriminatory in vitro methods for $\mathrm{QC}$, there is need for a better general understanding of these novel formulation types (16).

A rather uncommon dissolution method for QC of immediate-release tablet formulations is described in the final contribution to this special issue. The research paper "New Approach for the Application of USP Apparatus 3 in Dissolution Tests: Case Studies of Three Antihypertensive Immediate-Release Tablets" describes a method where the reciprocating cylinder apparatus (USP apparatus 3), an apparatus that was mainly established for in vitro drug release experiments for ER formulations, was successfully applied in designing dissolution methods for oral tablet formulations of three antihypertensive drugs with different solubilities. For the dosage forms screened in this study USP apparatus 3based methods presented with a higher robustness than a standard paddle (USP apparatus 2) method (17). This case example shows that even though there is currently a huge interest for developing bio-predictive methods, the development of QC methods based on compendial dissolution equipment is also still a challenge.

\section{REFERENCES}

1. Noyes AA, Whitney WR. The rate of solution of solid substances in their own solutions. J Am Chem Soc. 1897;19:930-4.

2. Gray VA. Power of the dissolution test in distinguishing a change in dosage form critical quality attributes. AAPS PharmSciTech. 2018;19(8):3328-32 Epub 2018/10/24.

3. Klein S. The use of biorelevant dissolution media to forecast the in vivo performance of a drug. AAPS J. 2010;12(3):397-406.

4. Garbacz G, Klein S. Dissolution testing of oral modified-release dosage forms. J Pharm Pharmacol. 2012;64(7):944-68 Epub 2012/06/13.

5. Koziolek M, Kostewicz E, Vertzoni M. Physiological considerations and in vitro strategies for evaluating the influence of food on drug release from extended-release formulations. AAPS PharmSciTech. 2018;19(7):2885-97 Epub 2018/08/30.

6. Schneider F, Hoppe M, Koziolek M, Weitschies W. Influence of postprandial intragastric pressures on drug release from gastroretentive dosage forms. AAPS PharmSciTech. 2018;19(7):2843-50 Epub 2018/05/31.

7. Felicijan T, Pislar M, Vene K, Bogataj M. The influence of simulated fasted gastrointestinal $\mathrm{pH}$ profiles on diclofenac sodium dissolution in a glass-bead flow-through system. AAPS PharmSciTech. 2018;19(7):2875-84 Epub 2018/08/29.

8. Karkossa F, Klein S. A bio-predictive in vitro comparison of oral locally-acting mesalazine formulations by a novel dissolution model for assessing intraluminal drug release in individual subjects. J Pharm Sci. 2018. Epub 2018/03/03;107:1680-9.

9. Karkossa F, Klein S. Individualized in vitro and in silico methods for predicting in vivo performance of enteric-coated tablets containing a narrow therapeutic index drug. Eur J Pharm Biopharm. 2019;135:13-24 Epub 2018/12/12.

10. Kesisoglou F, Vertzoni M, Reppas C. Physiologically based absorption modeling of salts of weak bases based on data in hypochlorhydric and achlorhydric biorelevant media. AAPS PharmSciTech. 2018;19(7):2851-8 Epub 2018/06/07.

11. Speer I, Preis M, Breitkreutz J. Novel dissolution method for oral film preparations with modified release properties. AAPS PharmSciTech. 2018;20(1):7 Epub 2018/12/19.

12. Pruessmann K, Wentzlaff M, Schilling R, Seidlitz A. Influence of dissolution vessel geometry and dissolution medium on in vitro dissolution behaviour of triamterene-coated model stents in different test setups. AAPS PharmSciTech. 2019;20(1):27 Epub 2019/01/04.

13. D'Arcy DM, Persoons T. Understanding the potential for dissolution simulation to explore the effects of medium viscosity on particulate dissolution. AAPS PharmSciTech. 2019;20(2):47 Epub 2019/01/09.

14. Long CM, Tang K, Chokshi H, Fotaki N. Surface dissolution UV imaging for investigation of dissolution of poorly soluble drugs and their amorphous formulation. AAPS PharmSciTech. 2019;20(3):113 Epub 2019/02/15.

15. Alvebratt C, Cheung O, Stromme M, Bergstrom CAS. A modified in situ method to determine release from a complex drug carrier in particle-rich suspensions. AAPS PharmSciTech. 2018;19(7):2859-65 Epub 2018/06/08.

16. Sheshala R, Anuar NK, Abu Samah NH, Wong TW. In vitro drug dissolution/permeation testing of nanocarriers for skin application: a comprehensive review. AAPS PharmSciTech. 2019;20(5):164 Epub 2019/04/18.

17. Espindola B, Bortolon FF, Pinto JMO, Pezzini BR, Stulzer HK. New approach for the application of USP apparatus 3 in dissolution tests: case studies of three antihypertensive immediate-release tablets. AAPS PharmSciTech. 2018;19(7):2866-74 Epub 2018/06/24.

Publisher's Note Springer Nature remains neutral with regard to jurisdictional claims in published maps and institutional affiliations. 